\title{
Cogan syndrome
}

INSERM

\section{Source}

INSERM. (1999). Orphanet: an online rare disease and orphan drug data base. Cogan

syndrome. ORPHA:1467

Cogan syndrome (CS) is a rare autoimmune disorder of unknown origin characterized by inflammatory ocular disease (mainly interstitial keratitis) and vestibulo-auditory manifestations (mainly acute onset hearing loss, tinnitus and vertigo), in the setting of a negative work-up for syphilis, with a variable risk of developing into a systemic disease. Systemic manifestations may occur in more than $70 \%$ of cases. 